\title{
ILCEA
}

Revue de l'Institut des langues et cultures

d'Europe, Amérique, Afrique, Asie et Australie

$12 \mid 2010$

La FASP : dix ans après...

\section{Quand la science enquête : imaginaires \& représentations de la FASP criminalistique}

\section{Shaeda Isani}

\section{OpenEdition \\ Journals}

Édition électronique

URL : http://journals.openedition.org/ilcea/583

DOI : 10.4000/ilcea.583

ISSN : 2101-0609

Éditeur

UGA Éditions/Université Grenoble Alpes

Édition imprimée

ISBN : 978-2-84310-180-9

ISSN : 1639-6073

Référence électronique

Shaeda Isani, «Quand la science enquête : imaginaires \& représentations de la FASP criminalistique », ILCEA [En ligne], 12 | 2010, mis en ligne le 29 septembre 2010, consulté le 21 avril 2019. URL : http:// journals.openedition.org/ilcea/583 ; DOI : 10.4000/ilcea.583

Ce document a été généré automatiquement le 21 avril 2019

(C) ILCEA 


\title{
Quand la science enquête : imaginaires \& représentations de la FASP criminalistique
}

\author{
Shaeda Isani
}

\section{Introduction : à propos de la FASP}

1 En 1999, Michel Petit identifie et codifie un «nouveau " genre de littérature populaire anglophone, auquel il donne le nom de «fiction à substrat professionnel» ou FASP. Si les romans de la FASP sont essentiellement des "thrillers», ils se démarquent par leur substrat professionnel, caractéristique définitoire qui renvoie à l'environnement professionnel ou spécialisé dans lequel l'imaginaire auctorial puise son inspiration, générant ainsi ce que Petit appelle « le thriller spécialisé » (1999, p. 194).

C'est une investigation présentée d'un point de vue qui est celui du milieu professionnel du personnage qui la conduit. Il en oriente les péripéties à partir de sa compétence professionnelle spécifique [...]. Il résulte de ce qui a été dit de la profession du personnage principal et du point de départ de l'histoire que l'intrigue est, dans la FASP, essentiellement de nature endogène : elle se forme à l'intérieur du réel professionnel qui l'engendre. Elle accorde en ce sens une large place à la représentation des divers éléments qui composent ce réel, personnes, objets, procédures. (1999, p. 195-196)

Les débuts de la FASP se décèlent dans le romanesque mais aujourd'hui le genre est largement présent dans le cinéma (la FASP cinématographique) et encore plus à la télévision (la FASP télévisuelle), et existe également en bande dessinée (la FASP bande dessinée). Les domaines professionnels ou spécialisés qui servent de substrat professionnel sont très variés et incluent la FASP juridique, sans doute le domaine le plus dominant du genre dont des auteurs tels que John Grisham, David Baldacci, Steve Martini et John Mortimer sont bien connus, la FASP médicale à travers les romans et séries télévisuelles de Michael Crichton, Robin Cook et Terry Gerritson, la FASP techno-militaire telle qu'illustrée par les romans de Tom Clancy, la FASP de l'art que représentent les 
romans d'Iain Pears et Neil Griffiths, ou encore la FASP journalisme à travers les romans de Stieg Larsson et Denise Mina.

\section{Substrat ou adstrat professionnel ?}

3 Il existe de nombreuses œuvres de fiction qui s'appuient sur un environnement professionnel sans pour autant relever de la FASP. À cet égard, il convient d'évoquer la distinction faite par Michel Petit entre substrat et adstrat professionnels. Pour ce qui concerne le premier, pour aspirer au statut de substrat professionnel, l'intrigue, la caractérisation et le dénouement de l'œuvre sont ancrés dans l'environnement professionnel et mis en fiction de manière à en être indissociables : le protagoniste évolue dans un milieu professionnel dont il est issu et qui est crucial au dénouement. Tel est le cas dans le premier roman de John Grisham, une FASP juridique intitulée A Time to Kill (1989) où l'avocat-protagoniste travaille la défense de son client, accusé d'assassinat, dans son cabinet, devant la cour, avec ses confrères et assistants, construisant sa stratégie de défense sur une loi qui traverse les péripéties du récit narratif.

Dans les cas où un tel degré d'osmose entre la trame fictionnelle et le domaine professionnel n'existerait pas, Michel Petit emprunte à la linguistique le terme d'adstrat ${ }^{1}$ professionnel, qu'il explique comme suit :

Outre les caractéristiques habituelles du thriller, les thrillers spécialisés de la FASP sont donc des romans qui conduisent le lecteur, à un rythme soutenu, au cœur même de milieux socio-professionnels qui ne sont pas les siens. Ceci ne paraît toutefois, en soi, pas caractéristique de la FASP. Il est clair en effet que toute œuvre de fiction se déroule dans un cadre particulier, que les personnages appartiennent à un certain milieu, etc. Dans le roman policier par exemple l'enquêteur est ainsi conduit à aborder un jour les milieux du théâtre, un autre ceux des marchands d'art, etc. Nous pensons qu'il ne s'agit pas là d'un véritable substrat professionnel au sens où nous l'entendons, et nous proposerons de parler plutôt, dans ce cas, d'adstrat professionnel.» (1999, p. 196)

Dans certaines œuvres, il s'agira d'un adstrat professionnel fort où le milieu professionnel, s'il ne représente pas la dynamique sur laquelle repose l'imaginaire du protagoniste, de l'intrigue et du dénouement, joue néanmoins un rôle actif et définitoire dans l'évolution du récit narratif. Le célèbre roman de Tom Wolfe The Bonfire of the Vanities (1987), se situe dans cette catégorie. Pour Michel Van der Yeught, il ne peut pas s'agir d'une FASP à proprement parler, puisque «le ressort romanesque [...] ne se situe pas dans le milieu professionnel, mais dans la déchéance de l'ex-« maître de l'univers » Sherman McCoy. [Ce récit], bien que situé dans l'environnement boursier de Wall Street, ne relève pas de la FASP.» (2009, p. 79) Le milieu de la bourse est néanmoins, omniprésent non seulement au niveau du locus, des personnages et des représentations mais également en tant qu'ethos culturel qui contribue à la déchéance du protagoniste.

6 Inversement, il s'agira d'un adstrat professionnel faible dans d'autres cas où le milieu professionnel est présent essentiellement à titre de décor ou de simple identifiant pour le protagoniste. Tel est le cas dans le roman de Louis Begley, About Schmidt (1996) où, bien que le paratexte à la quatrième de la couverture commence par les mots, "Proud, traditional, and impeccably organized, Albert Schmidt is a buttoned-down lawyer of the old school. ", l'utilisation du présent s'avère trompeuse car le roman tourne essentiellement autour de la retraite du protagoniste, sa vie active en tant qu'avocat étant relégué au référentiel et à l'anecdotal. 
7 Il convient de rappeler ici que d'autres œuvres de la FASP entretiennent des rapports substrat/adstrat plus complexes. C'est notamment le cas de la FASP journalisme qui est définie par la nature symbiotique du lien entre le substrat professionnel (le journalisme) et l'adstrat professionnel dont celui-ci est tributaire pour exister (Isani, 2009a). Ainsi, la FASP journalisme de Denise Mina s'appuie sur l'enquête policière, celle de Stieg Larsson sur le monde politique et celle de Liza Marklund intitulée The Bomber (1998) sur l'organisation des jeux olympiques de Stockholm.

8 The Bonfire of the Vanities semble, à plusieurs égards, annoncer l'évolution du statut de l'adstrat professionnel dans la mesure où s'entremêlent dans ce roman différents adstrats professionnels pour former une mosaïque interdisciplinaire composée des domaines de la finance, de la justice et du journalisme. À cet égard, John Grisham, auteur habituellement associé à la FASP juridique, a quelque peu désorienté son lectorat avec la publication de The Last Juror (2004), une FASP dans lequel le droit est réduit au statut d'un adstrat professionnel tributaire d'un substrat professionnel journalistique. Jean-Pierre Charpy identifie la même tendance chez Robin Cook, auteur de FASP médicale, dans son roman Crisis (2006) où le substrat médical cohabite avec le substrat juridique :

En 2006, le père fondateur du roman à suspense à dominante médicale, R. Cook, publie Crisis, une forme hybride protéiforme reposant sur l'osmose de trois genres spécialisés existants. En effet, on y trouve l'adstrat lexical spécialisé des romans de la FASP juridique aux côtés de l'adstrat spécialisé de la médecine légale et du substrat professionnel de la médecine. (2010, p. 59)

Dans son analyse de l'émergence d'un nouveau sous-genre, la FASP écologique, Camille Biros (2010) observe le même phénomène par rapport à ce domaine qui, de par son essence interdisciplinaire, regroupe des acteurs d'origines professionnelles hétérogènes (scientifiques, militants, responsables politiques, entrepreneurs, voire sportifs). Ainsi, la notion d'une FASP définie par une dominante professionnelle monolithique commence à céder du terrain en faveur d'une composite d'adstrats professionnels ou spécialisés et correspond, sans doute, également à la contrainte éditoriale qu'a tout auteur de fiction populaire de satisfaire à un lectorat dont la fidélité se traduit par des exigences croissante vis-à-vis du substrat professionnel. (Isani, 2009b)

Une autre évolution générique qui a marqué la FASP au cours de la décennie qui s'est écoulée depuis sa codification en tant que genre à part entière, est l'émergence de nouveaux sous-genres dont le substrat professionnel est souvent le reflet des préoccupations sociétales contemporaines, tel que la FASP écologique ou l'« écothriller " évoqué supra (Biros, 2010), ou encore la FASP criminalistique qui fait l'objet de cette étude.

\section{À propos des sciences criminalistiques}

11 L'essor des sciences qu'a connu le xix siècle a imprégné toutes les sphères de la vie professionnelle, y compris celles de l'enquête policière où un profond changement est apparu dans la façon d'appréhender le criminel, comme le souligne Patrick Pesnot dans son ouvrage, Les Détectives de l'impossible:

Désormais la police serait scientifique, la déduction triompherait de la conviction et de l'intuition, l'administration de la preuve confondrait le mensonge et les faux témoignages. Le détective et le savant étaient promis à une collaboration obligée, souvent passionnée, toujours tumultueuse. Le fait divers rencontrait la science. Ils ne devaient plus se quitter. Les hommes en blouse blanche, médecins, chimistes, 
biologistes, physiciens, informaticiens allaient peu à peu devenir les indispensables auxiliaires de l'enquêteur du terrain. Leur activité, leurs tâtonnements, leurs découvertes et parfois leurs erreurs seraient maintenant associés aux affaires criminelles les plus retentissantes de notre temps. $(1999$, p. 15)

Intrinsèquement interdisciplinaire, la criminalistique est un domaine qui s'étend de manière constante vers de nouveaux horizons. Située à la croisée du droit et des sciences dures (de l'étude des éclaboussures de sang à l'analyse de l'ADN, en passant par la balistique, la toxicologie, la graphologie, l'analyse documentaire, l'odontologie, l'anthropologie, l'entomologie, l'histologie, etc.), la criminalistique fait appel aussi à des disciplines relevant du domaine de l'art (la sculpture craniofaciale, ou encore l'expert en art, très sollicité dans le vaste domaine du vol et de la fraude des œuvres d'art), voire à la linguistique, un domaine en pleine croissance que Malcolm Coulthard définit comme suit dans The Linguistics Encyclopedia :

In 1968, Jan Svartvik published The Evans Statements: A Case for Forensics Linguistics, in which he demonstrated that disputed parts of a series of statements which had been dictated to Timothy Evans by police officers, and which incriminated him in the murder of his wife, had a grammatical style measurably different from that of uncontested parts of the statements. A new discipline was born... [...] Forensic linguists help courts to answer three questions about a text - What does it say? What does it mean? Who wrote/typed/authored it? (2004, p. 139-140)

13 Si aujourd'hui les sciences criminalistiques sont un domaine largement dominé par les Anglo-Saxons à la fois dans la vie réelle et sur l'écran, il conviendrait de rappeler que les origines en sont bien françaises. L'un des premiers pionniers de l'approche scientifique en matière d'enquête policière est Alphonse Bertillon (1853-1914) qui fonde le premier laboratoire de police scientifique et invente l'anthropométrie judiciaire comme système d'identification des criminels. Malgré ses avancées, les spécialistes s'accordent pour dire que le "père spirituel» de cette discipline est un prodigieux scientifique lyonnais, Edmond Locard (1877-1966), dont la double formation en médecine et en droit le situe au croisement des deux disciplines définitoires des sciences criminalistiques. C'est à Edmond Locard que nous devons le principe fondateur de cette discipline, appelé "principe d'échange de Locard » qui affirme qu'« on ne peut aller et revenir d'un endroit, entrer et sortir d'une pièce sans apporter et déposer quelque chose de soi, sans emporter et prendre quelque chose qui se trouvait auparavant dans l'endroit ou la pièce. » ([En ligne] $<$ http://sciencesforensiques.com>), principe que Jacques Fombonne, professeur de droit, explicite ainsi dans son ouvrage intitulé La criminalistique :

[Il existe un] principe d'échange permanent entre l'homme et le milieu ambiant, et de l'accélération de ce phénomène avec la violence inhérente à l'acte criminel. Cela signifie en pratique qu'un criminel entrant par exemple dans un appartement va apporter avec lui les éléments dont il est le générateur ou le vecteur: traces digitales, empreintes de pas, poussières collées sous ses chaussures, cheveux perdus, fibres de vêtements, particules de sang laissées sous les ongles de sa victime... Simultanément, il emportera malgré lui, à l'issue de son forfait, les particules de la vitre qu'il aura dû briser, les microscopiques taches de sang de sa victime, des fibres de la moquette... brève, l'ensemble des éléments qui va prouver sa présence sur les lieux. (1996, p. 46)

14 Il apparaît ainsi clair que la criminalistique s'intéresse non pas aux hommes mais aux "traces» qu'ils laissent ou qu'ils emportent. Dans une approche sémiotique, cette démarche peut être assimilée à la textualisation des signes laissés par le passage de l'homme, des éléments de silence que la science va lire, décoder et interpréter dans le cadre d'une lecture identitaire souvent dénonciatrice. 

partie dû au fait que les sciences criminalistiques n'existent pas en tant que discipline en soi mais regroupent plusieurs disciplines appliquées à l'investigation criminelle dans le but d'apporter des preuves objectives, d'inculper ou disculper, dans le procès pénal et civil, comme l'explique l'Encyclopédie Universalis en ligne :

La criminalistique est l'art et la science de découvrir, d'analyser et d'identifier les indices matériels des faits; sa méthodologie et sa technique sont essentiellement consacrées à la découverte du fait judiciaire et à l'identification de son auteur. Pour atteindre ce but, la criminalistique fait largement appel aux techniques et aux méthodes des sciences fondamentales et appliquées : sciences naturelles, physiques, chimiques, biologiques, et, dans ce dernier domaine, la médecine légale apporte une contribution importante. Son mode de raisonnement est différent de celui des sciences dont elle dérive, car elle s'occupe seulement de découvrir les effets d'un acte délictueux. Multidisciplinaire par essence, la criminalistique doit son développement à celui des sciences. Son champ d'action est aussi vaste que celui des problèmes qui peuvent être soulevés, tels que l'identification des personnes en matière pénale, l'identification des traces humaines susceptibles d'être examinées par les procédés de laboratoire, l'identification des traces et des taches non biologiques et des objets dans les situations d'intérêt juridico-pénal. <http:// www.universalis.fr>, consulté le 18/06/2009.

La discipline de la criminalistique souffre d'une certaine méconnaissance auprès du grand public européen, phénomène que plusieurs raisons expliquent, dont les plus importantes sont, sans doute, l'absence d'une définition formelle de la discipline et les différences structurelles qui existent entre l'organisation des sciences criminalistiques en Europe et celle des aires relevant de la culture de common law. À ce dernier élément, il faudrait rajouter le fait paradoxal qu'en vertu de son exposition aux séries télévisuelles américaines et de l'absence de connaissances sur son propre système, le public européen s'approprie le système américain comme faisant partie de sa propre culture juridique (Isani, à paraître en 2010).

Pour Jacques Fombonne, l'organisation de la criminalistique en France - et par extension, dans la plupart des aires juridiques relevant du droit civil - « est la plus complexe et la moins logique » (1996, p. 9). Pour simplifier, il conviendrait de dire que la criminalistique en France et dans d'autres pays de tradition civiliste est divisée en deux entités distinctes, la police technique et la police scientifique. La police technique est, d'après cet auteur, une notion typiquement civiliste qui n'apparaît jamais dans la littérature anglo-saxonne et qu'il définit comme, "l'activité diligentée, avant tout autre, sur les lieux d'une infraction pénale et visant à la révélation, au rassemblement et à la conservation inventoriée, de toutes les manifestations matérielles de l'acte incriminé, en vue de leur exploitation scientifique future » $(1996$, p. 11). Ainsi, pour résumer, la police technique se charge des opérations dites "foraines ", c'est-à-dire, les actes effectués in situ, sur le lieu du crime.

18 La police scientifique, quant à elle, est l'entité qui prend la suite logique et successive de la police technique pour exploiter et convertir les indices ainsi recueillis en preuve expertale, c'est-à-dire, « l'activité diligentée en aval de la police technique et qui consiste au sein d'une structure permanente telle qu'un laboratoire, à exploiter les indices matériels, dans le but d'en obtenir une preuve exploitable au cours de l'enquête pénale. » (Fombonne, 1996, p. 12) Malgré la nature essentiellement sédentaire et discrète de son travail, il y a au moins un déplacement souvent très médiatisé qui expose au grand jour la police scientifique, celui où son représentant est appelé à la barre pour répondre aux 
"questions judicieuses» des requérants ou, autrement dit, pour témoigner devant le tribunal en sa capacité d'« expert ».

À ce cloisonnement binaire vient s'ajouter le fait que certaines disciplines fondamentales de l'enquête judiciaire telles que la médicine légale, la toxicologie et la psychiatrie légale ne sont pas comprises dans la criminalistique. L'une des raisons avancées pour cette séparation viendrait du caractère « universitaire » de ces disciplines et du lien ancestral entre, par exemple, la médecine légale et l'instruction judiciaire qui contraste avec l'aspect relativement "technique » et récent des sciences criminalistiques telles que la balistique, l'odontologie, les empreintes digitales, la recherche de l'ADN, etc.

La criminalistique s'organise de manière sensiblement différente dans les aires relevant de la culture de la common law. Aux États-Unis, il semblerait que le cloisonnement technique/scientifique soit bien moins marqué et que ce soit une équipe «intégrée » qui intervienne lorsque survient un fait judiciaire. Autrement dit, ce sont les mêmes professionnels qui effectuent les prélèvements in situ et les analysent ensuite au laboratoire scientifique. Comme le précise Jacques Fombonne, "s'y intègrent naturellement tous les actes de la recherche, depuis la première photographie des lieux jusqu'à la signature du rapport d'expertise, en passant par les conclusions du médecin légiste ou du psychiatre.» (1996, p. 21) Telle est effectivement la représentation présentée de ce milieu professionnel dans la série télévisuelle CSI Miami où policiers et scientifiques font partie de la même équipe et s'acquittent des deux types de tâches.

21 Au Royaume-Uni, une large partie des services criminalistiques s'organise autour de l'ancienne institution du coroner et de son inquest dont la fonction essentielle est d'établir la cause du décès. L'organisation de cette institution s'approche quelque peu de celui des pays de droit civil dans la mesure où les SOCOs (scene of crime officers) sont chargés du prélèvement des "traces" et des experts, souvent des scientifiques extérieurs à la juridiction du Coroner, sont chargés de l'analyse, comme le reflète le seul forensic thriller écrit par P.D. James, Death of an Expert Witness (1977), où le laboratoire scientifique en question travaille pour la police, mais indépendamment de celle-ci.

La méconnaissance du grand public européen au sujet du véritable fonctionnement des services criminalistiques est aggravée par les perceptions erronées formées à partir des séries télévisuelles américaines. L'évolution de la police scientifique française contribue également à accroître cette confusion : s'il pouvait arriver par le passé que la police scientifique se déplaçât sur le lieu du crime pour travailler avec la police technique dans le cas des circonstances exceptionnelles, aujourd'hui de plus en plus, c'est la science qui se déplace grâce aux unités mobiles équipées des dernières technologies - et c'est ainsi que la fiction des séries télévisuelles américaines devient la réalité de la police scientifique française.

\section{Quelques questions de traduction}

23 La discipline qui fait l'objet de cette étude présente un certain nombre de problèmes dans le domaine de la traduction, notamment par rapport à la traduction de l'anglais vers le français où il existe un état diglossique entre deux termes, forensics ${ }^{2}$ et criminalistics.

The Shorter Oxford English Dictionary on Historical Principles nous enseigne que le terme forensic est attesté comme adjectif depuis 1659 et désigne tout ce qui à trait à ou est utilisé pour ou par le tribunal : «Pertaining to, or connected with, or used in courts of law; suitable or 
analogous to pleading in court. » (1973, p. 788). En revanche, le terme criminalistics n'y est pas attesté. D'autres sources l'attestent (Katherine Ramsland, 2006, p. 276) ou encore la source en ligne enotes qui confirme la confusion terminologique qui semble régner dans ce domaine :

Criminalistics is one subdivision of forensic sciences. The terms criminalistics and forensic sciences are often confused and used interchangeably. [...] It is very difficult to provide an exact definition of criminalistics, or the extent of its application, as it varies from one location or country to another. However, the American Board of Criminalistics defines criminalistics as "that profession and scientific discipline directed to the recognition, identification, individualization, and evaluation of physical evidence by application of the physical and natural sciences to law-sciences matters." The California Association of Criminalistics provides a slightly different definition [...]. These definitions are very similar to the ones used for forensic sciences, as both disciplines have as a goal to provide scientific analysis of evidence for the legal system. <http://www.enotes.com/...>, consulté le $18 / 06 / 2009$.

La fiction se fait également l'écho de ce débat lexicologique autour de ces deux termes spécialisés, criminalistique et forensique, comme en témoigne cet extrait du roman The Bone Collector de Jeffrey Deaver qui y ajoute dimension conative intéressante :

« How's the world's foremost criminalist?» Cooper asked him.Rhyme scoffed good-naturedly. The title had been bestowed on him by the press some years ago, after the surprising news that the FBI had selected him - a city cop - as adviser in putting together PERT, their Physical Evidence Response Team. Not satisfied with "forensic scientist" or "forensic specialist", reporters dubbed Rhyme a "criminalist".The word had actually been around for years, first applied in the United States to the legendary Paul Leland Kirk, who ran the UC Berkeley School of Criminology. [...] The handle had recently become chic, and when techs around the country sidled up to blondes at cocktail parties now they described themselves as criminalists, not forensic scientists. (1998, p. 72)

26 La traduction en français du terme forensic sciences présente des difficultés liées essentiellement à des facteurs historiques et structurels. Comme déjà évoqué, la médecine légale, dont les activités dans le domaine thanatologique et de l'acte d'autopsie sont mieux connues que les activités cliniques, fut pendant longtemps, la seule discipline à être admise comme faisant autorité auprès du magistrat. Le caractère traditionnel et exclusif de ce partenariat entre la justice et la médecine légale explique le recours à ce terme pour désigner tous les aspects scientifiques de la procédure judiciaire, comme en témoignent encore aujourd'hui certaines traductions proposées par le Dictionnaire Juridique/Law Dictionary (2004) réalisé conjointement par Harrap's et Dalloz où l'équivalent proposé pour forensics department est "service médicolégal », une traduction manifestement insatisfaisante dans le cas d'un laboratoire qui réalise des analyses concernant les empreintes de pneus ou encore la trajectoire des balles.

Dans les années 1970, Josette Rey-Debove et Alain Rey valident le terme "criminalistique», attesté depuis 1907, comme "science étudiant les techniques de recherche de la preuve des crimes et de leurs auteurs et les procédés d'investigation policière propres à les mettre en pratique.» $(2005$, p. 594$)$ et nous noterons l'assimilation qui s'opère au niveau lexicographique entre les deux entités de la criminalistique.

La traduction se complique lorsque le terme est employé comme adjectif: alors que l'anglais parle aisément de forensic expert, forensic orthodentist, forensic accountant, forensic anthropologist, le français peine à trouver un équivalent à la fois clair et ergonomique - «expert criminalistique»? - ce qui explique peut-être la persistance du terme « médicolégal ». Ainsi, le Dictionnaire Juridique/Law Dictionary (2004) donne un exemple où 
"forensic tests showed him to be the killer» est rendu par «les tests médico-légaux ont prouvé qu'il était l'assassin » (2004, p. 53).

Les nombreuses séries télévisuelles en anglais portant sur ce domaine présentent une piste d'exploration intéressante à cet égard : comment les professionnels traduisent-ils le terme forensic sans qu'il soit "lost in translation"? La première interrogation porte naturellement sur la façon dont le problème est traité dans le cadre d'une série française, R.I.S Police Scientifique. Il s'agit, en fait, d'une série qui s'inspire d'une série italienne, RIS Delitti Imperfetti fondée sur les exploits d'une division des carabiniers, Ricerche $e$ Investigazioni Scientifiche. Les producteurs ont su contourner le problème en calquant le titre italien sous forme d'acronyme - sans doute en mimétisme de la série américaine CSI - suivi par l'apposition "police scientifique» qui a le mérite d'être accrocheur et transparent même si seulement partiellement en phase avec la réalité.

C'est en analysant le travail des traducteurs des séries télévisuelles anglophones, que nous gagnons quelques indices précieux relatifs à cette problématique très spécifique. Les traducteurs de la série américaine CSI (Crime Scene Investigation) ont choisi de contourner la difficulté en recourant à des stratégies de sous-traduction par l'emploi d'un générique, traduisant ainsi le titre tout simplement par Les Experts. En revanche, les traducteurs des séries britanniques - Inspector Morse, Lewis et Midsomer Murders - semblent avoir trouvé la note heureuse en traduisant forensic experts par « les experts judiciaires ».

Le problème de la traduction vers le français s'étend au-delà des frontières de la France. Si le français québécois valide également le terme de « criminalistique ", le français suisse apporte une précision supplémentaire, comme l'indique l'éminent Institut de Police Scientifique et de Criminologie de l'université de Lausanne (pionnier du domaine et encore aujourd'hui un haut lieu des sciences criminologiques) sur le site de la Chancellerie fédérale de la Confédération suisse :

La criminalistique désigne l'exploitation des indices dans l'investigation criminelle, alors que la police scientifique définit un domaine encore plus restreint des sciences forensiques: les techniques appliquées aux enquêtes policières uniquement. <http://www.bk.admin.ch/..., consulté le 04/05/2009.

D'après cette distinction, le travail de la police scientifique relève des «sciences forensiques », qui seraient l'une des composantes du domaine de la criminalistique. Par ailleurs, cet Institut paraît être favorable à la généralisation du terme "forensique », en arguant un pragmatisme transculturel :

L'adjectif forensique (qui appartient à la cour de justice, qui relève du domaine de la justice) est un néologisme. Il vient du latin forum (place publique, lieu du jugement dans l'Antiquité). Il fait partie du vocabulaire de pratiquement toutes les langues qui nous sont proches comme l'allemand, l'italien et l'anglais, mais son usage est récent en français. <http://www.bk.admin.ch/...>, consulté le 04/05/2009.

Ces réflexions nous conduisent à nous interroger sur l'appellation du sous-genre de la FASP. Si forensic FASP sied bien en anglais, quid du français? Faut-il, dans le même esprit de pragmatisme transculturel prôné par l'Institut de Police Scientifique et de Criminologie de l'Université de Lausanne, et malgré son aspect transgressif, opter pour «FASP forensique »? Ou, dans une approche plus orthodoxe, retenir le terme attesté avec «FASP Criminalistique»? Bien que les deux termes sonnent aussi mal aux oreilles du lectorat de masse, le terme " criminalistique ", pour aussi peu familier qu'il soit, possède néanmoins le mérite d'une transparence que n'a pas «forensique ». Ainsi, dans le cadre de cette étude, nous proposons de référer à ce sous-genre de la FASP comme la FASP 
criminalistique, choix appuyé par le fait que le terme criminalistics est attesté également dans d'autres langues comme l'anglais et l'allemand.

\section{À propos de la FASP criminalistique}

\section{Émergence d'un nouveau genre} nouveaux domaines scientifiques applicables à l'enquête judiciaire, tels que la photographie, la polygraphie (détecteur de mensonge), la dactyloscopie (la classification des empreintes digitales), l'autopsie, l'odontologie, la balistique, la sérologie, etc. La littérature se fait le reflet de cette fusion entre science et justice et le $\mathrm{xIX}^{\mathrm{e}}$ siècle en Angleterre et aux États-Unis voit des écrivains comme Edgar Allan Poe, Wilkie Collins, Nathaniel Hawthorne, Arthur Conan Doyle, Mark Twain, Joseph Conrad, etc., exprimer l'imaginaire auctorial à travers des detective fiction novels où l'intrigue fait une large place aux innovations scientifiques et techniques (Thomas, 1999).

C'est sans doute le célébrissime personnage imaginé par Sir Arthur Conan Doyle - luimême diplômé de médecine et d'ophtalmologie et passionné de photographie - qui incarne ce genre littéraire qui célèbre la fusion entre l'enquête criminelle et la science, comme le proclame Patrick Pensot sur la $4^{\mathrm{e}}$ de couverture de son ouvrage: « $\mathrm{Au}$ commencement était Sherlock Holmes... Jamais une science, la criminalistique, ne s'est autant identifiée à une aventure romanesque. » À cet égard, nous notons dans le récit le croisement de personnages scientifiques contemporains et de personnages fictionnels : Alphonse Bertillon, pionnier dans le domaine de l'identification criminalistique, est à plusieurs reprises explicitement évoqué par Sherlock Holmes et inversement, Edmond Locard conseille à ses étudiants de lire A Study in Scarlet (1887) de Conan Doyle pour mieux comprendre la science de la criminalistique. Cette fusion fiction-réel perdure jusqu'à nos jours, comme en témoigne le livre de Michel Mazévet intitulé Edmond Locard: Le Sherlock Holmes français, publié en 2006.

Les années 1990 voient l'essor de la FASP criminalistique telle que nous l'entendons aujourd'hui : il ne s'agit plus d'un protagoniste individuel dont l'approche scientifique rend singulier, mais d'un substrat professionnel à part entière qui relève d'un environnement spécialisé où la science et les technologies de pointe constituent les outils de travail quotidiens des professionnels mis en fiction. Pour la première fois, la technologie devient le point de mire et l'œil du spectateur est invité à se poser sur le microscope en même temps que celui du scientifique. Ce rapprochement fictionnel entre science et thriller connaît un succès immédiat créant ainsi la masse critique pour consolider la FASP criminalistique en tant que genre, comme en témoignent les longues listes de forensic et CSI fiction proposées par différents sites web dédiés au recensement des thrillers relevant de la FASP criminalistique ${ }^{3}$.

Deux auteurs sont particulièrement associés à la FASP criminalistique, Patricia Cornwell et Kathy Reichs. Le premier roman FASP à proprement parler, date sans doute de 1990 lorsque Patricia Cornwell, auteur américain, publie Postmortem, dans lequel le lectorat prend connaissance du milieu « forensique » fictionnel à travers Kay Scarpetta, médecin légiste, protagoniste récurrent des dix-huit romans écrits jusqu'à aujourd'hui. En 1998, l'auteur biculturel américain-québécois, Kathy Reichs, vient étoffer la FASP criminalistique en publiant Déjà Dead dans lequel elle présente Tempe Brennan, 
paléoanthropologue judiciaire et protagoniste de la douzaine de romans FASP criminalistique publiés jusqu'à aujourd'hui.

Bien que des séries télévisuelles à substrat professionnel criminalistique existent depuis un certain temps déjà - Quincy, M. E. aux États-Unis (1976-1983) et Silent Witness de la BBC (1996-2008), par exemple - ce n'est qu'en 2000 que la criminalistique crève littéralement l'écran avec la série télévisuelle américaine CSI Las Vegas. Le succès immédiat que connaît CSI Las Vegas - « la série la plus regardée au monde » - conduit à la création de CSI Miami en 2002 et CSI New York en 2004, suivies, en 2005, par l'adaptation au petit écran de la FASP criminalistique romanesque de Kathy Reichs, avec son personnage Tempe Brennan, dans une série télévisuelle intitulée Bones. Le succès de ces séries est dû à de multiples facteurs mais deux retiennent particulièrement notre attention dans le cadre de la FASP : un lectorat qui cherche à s'instruire tout en se divertissant et l'accès qu'accorde le substrat professionnel criminalistique au monde fascinant mais hermétique de la science, comme l'indique Sue Turnbull dans l'ouvrage collectif Reading CSI: Crime TV Under the Microscope :

CSI, I would argue, clearly participates in [...] an Enlightenment project in an era when, for most people, science is a discourse to which they have no access except through the popular medium of a television programme which not only tells but shows as well. (2007, p. 31)

\section{Évolution du genre}

39 Comme déjà évoqué, la criminalistique n'est pas une discipline monolithique mais un domaine composé de plusieurs disciplines très diversifiées. Cette caractéristique trouve son reflet fidèle dans la FASP criminalistique où le substrat professionnel s'articule autour de trois principaux axes professionnels, le domaine de l'enquête policière et les disciplines du droit et des sciences.

À l'instar de CSI Miami où les scientifiques sont des policiers - ce qui n'est pas toujours le cas dans la réalité - la FASP criminalistique tend à gommer les frontières entre l'enquête judiciaire et les disciplines scientifiques. Cette fusion entre le personnage du policier et celui du scientifique influe sur l'évolution du genre dans la mesure où, au niveau du récit, elle place les deux domaines professionnels en concurrence, conduisant ainsi à l'absence d'un substrat professionnel dominant.

\section{Le droit en tant qu'adstrat professionnel}

41 La discipline du droit plane sur tout le récit narratif, de manière plus ou moins implicite, par l'aspect thriller et enquête du genre. Sa présence est plus marquée en amont et à l'aval de l'intrigue. En amont de l'intrigue, le droit pèse de tout son poids sur les moyens et les processus mis en œuvre dans le prélèvement des précieuses «traces» définies par Edmond Locard, tout écart de la procédure d'identification, de transport et d'analyse de l'échantillon obtenu ("safe custody»), telle que définie par la loi pouvant entrainer l'invalidation de l'éventuelle preuve ainsi obtenue.

Le droit est aussi présent en aval de l'intrigue lorsque la fusion entre le droit et la science est mise en lumière par le sacre du scientifique en tant que témoin expert à la barre. Il s'agit d'un rôle largement exploité dans la fiction et tous se souviennent des passages et des scènes où l'expert en balistique trace la trajectoire d'une balle et disculpe ainsi l'accusé, ou encore comment tel autre expert parvient à lire les derniers mouvements de la victime ou de son meurtrier dans une éclaboussure de sang ${ }^{4}$. 
Malgré cela, il est clair que si le droit est un cadre référentiel omniprésent dans la FASP criminalistique, c'est la science qui prime au niveau de la dynamique narrative. Cette observation nous conduit à constater que l'une des caractéristiques définitoires de la FASP criminalistique est qu'elle déroge au principe canonique du substrat professionnel dominant pour se construire autour d'un composite d'adstrats professionnels étroitement enchevêtrés et définis par une interdépendance symbiotique. En cela la FASP criminalistique se situe comme annonciatrice de l'évolution évoquée supra où l'orthodoxie générique du substrat professionnel monolithique se voit petit à petit émiettée au profit d'une mosaïque d'adstrats professionnels.

\section{Montée des sciences criminalistiques en tant qu'adstrat professionnel}

La criminalistique ne peut, à elle seule, constituer le substrat professionnel d'une œuvre de fiction. En revanche, elle est de plus en plus mise en valeur en tant qu'adstrat professionnel, en partie sans doute comme stratégie éditoriale destinée à exploiter la vague de popularité que connaissent les sciences criminalistiques depuis les années 2000. Ainsi, nous observons que Tess Gerritson, auteur de nombreuses FASP médicales, opère un genre shift en 2001 avec une nouvelle série basée sur un tandem entre deux professionnelles, l'une détective et l'autre médecin légiste.

D'autres auteurs de FASP policière ou du prétoire, tout en restant fidèles au genre initial, exploitent l'engouement pour la scientificité fictionnelle en renforçant le rôle de la criminalistique dans le récit narratif. L'une des techniques narratives couramment utilisées à cet effet consiste à mettre en avant le rôle d'un professionnel de la criminalistique en tant que "témoin expert» devant le tribunal. Ainsi, Steve Martini, auteur de FASP judiciaire, puise dans le domaine très technique de la balistique pour son roman Double Tap (2005) dont l'un des points forts est le témoignage de l'expert en balistique à la barre :

"Can you tell the jury the purposes behind the design of frangible bullets?"

"There are several purposes and several different kinds of frangible bullets. Usually they are subsonic rounds: that is, the velocity of the bullet is designed and intended to remain below the speed of sound, roughly a thousand feet per second." [...]

"Go on, tell us the purposes of the round."

"A forty-five-caliber automatic, such as the murder weapon in this case, is generally - unless it's a special manufacture - in the nine-hundred-foot-per-second range in terms of bullet velocity. It is considered a large-bore pistol. Frangible rounds are a perfect fit for such a weapon. They could be used for target shooting where, for reasons of safety because of protocols of close-in fire training, you want to avoid ricochet. They are also used by law enforcement in certain hostage-rescue situations - on board airliners, for example. The frangible round is designed to fragment whenever it strikes something harder than itself, so it avoids overpenetration."

"What is that? Overpenetration. Explain to the jury if you could."

"In a hostage situation - say, where there are numerous innocent hostages - you would not want to shoot through your intended target, have your bullet pass through one of the hostage takers and hit an innocent victim. So a frangible round would be a good choice. The fragments would be absorbed, contained within the target. [...]" (2005, p. 283-284.)

C'est sans doute dans le cadre des séries télévisuelles policières produites pour le petit écran que la mise en valeur de la criminalistique comme adstrat professionnel est la plus frappante. Si elle en a toujours fait partie, le rôle de la criminalistique se restreignait jusqu'alors à celui du «figurant ». Un exemple qui illustre singulièrement cette évolution est les deux séries britanniques Inspector Morse (1987-2000) et la suite créée en 2006 
intitulée Lewis, du nom de l'adjoint du défunt Inspecteur Morse, devenu à son tour inspecteur à la ville d'Oxford. Dans la série Inspector Morse, la criminalistique est présente de manière ponctuelle et brève alors que dans la série Lewis, elle est promue au rang d'un rôle récurrent, avec mise en avant de toute la panoplie de la criminalistique et du médecin légiste, personnalisé par le personnage de Dr Laura Hobson, une jolie femme blonde. La même tendance peut être constatée dans les dernières saisons de la série Midsomer Murders (Inspecteur Barnaby en français) où les techniciens de la criminalistique et le médecin légiste, encore un personnage récurrent, s'invitent de manière quelque peu incongrue dans l'univers bucolique de Midsomer, un village fictif sans doute inspiré des romans d'Agatha Christie. Et finalement, aux États-Unis, la série américaine NCIS (2003), une FASP policière spécifique aux enquêtes au sein de la marine, est également conçue autour d'un adstrat professionnel criminalistique par le biais des deux personnages les plus marquants de la série, Abby la laborantine gothique et Ducky, le médecin légiste bavard d'origine écossaise.

\section{Représentations fictionnelles de la criminalistique}

47 L'un des principaux axes de recherche dans le domaine de la FASP porte sur les représentations et les perceptions qu'engendrent cette mise en fictions des professionnels et des milieux professionnels dans la littérature populaire. Dans l'axe de nos travaux sur les représentations fictionnelles de l'avocat américain (2005), du magistrat (2010) et du journaliste (2009a), et en partant d'une approche comparative entre les représentations fictionnelles de ce milieu et de ses professionnels dans la FASP criminalistique et dans la fiction qui a précédé l'émergence de ce sous-genre, nous proposons ici d'analyser les modes scriptural et visuel qui contribuent aux représentations fictionnelles de la police criminalistique à travers les portraits de trois personnages clés de la FASP criminalistique : le policier scientifique (forensic expert), le médecin légiste et... le cadavre.

\section{Le policier scientifique : la science en action}

48 Le scientifique fictionnel subsume les différentes catégories de personnel technique impliqué dans la criminalistique, qu'il s'agisse du technicien, du laborantin ou du scientifique. Dans la FASP policière traditionnelle où le rôle du policier domine celui du scientifique, ce dernier est représenté comme un personnage que rien ne distingue d'autres professionnels et la criminalistique est un domaine où les analyses prennent du temps, les résultats se font attendre et sont parfois erronés. Dans ce contexte, les limites du scientifique et de la science sont constamment évoquées, comme en témoigne cet extrait de Death of an Expert Witness, une FASP criminalistique pionnière écrite en 1977 par P. D. James, où s'affichent les réticences traditionnelles par rapport aux pouvoirs magiques des sciences criminalistiques :

He knew how little forensic scientists could do with only four hairs, but it was still possible that something could be learned. [...]

'I'd like you to have a look at these four hairs urgently for me. [...] I want to know, if possible, if the dark hairs came from the same head.'

'It's easier to say whether they don't. I can have a look under the microscope but doubt whether I can help you. Hair identification is never easy, and I can't hope to do much with only three samples. Apart from microscopic examination, we'd normally use mass 
spectrometry to try and identify differences in the trace elements, but even that isn't possible with three hairs. If these were submitted to me, I'd have to say that I couldn't give an opinion." (2005, p. 285) de Patricia Cornwell et de Kathy Reichs où les protagonistes sont des femmes hors normes qui sont non seulement belles et intelligentes, mais également capables d'exercer deux métiers exigeants dans deux villes, voire deux pays, différents, d'assumer des postes d'autorité et de responsabilité, et d'être des professionnelles scientifiquement respectées par leurs pairs. Cette image est rehaussée par l'astuce narrative qui consiste à faire découvrir la vérité par la femme scientifique à la place de la police judiciaire (traditionnellement de la gente masculine), officiellement chargée de l'enquête. criminalistique télévisuelle des séries américaines où les personnages du policier et du scientifique fusionnent pour générer un seul personnage qui relève les preuves indiciaires in situ, les analyse au laboratoire et mène à l'enquête. Le personnage du scientifique-enquêteur de la série télévisuelle est construit par le biais d'un dédoublement de l'identité professionnelle. Chaque persona est soigneusement mise en scène et balisée par des marqueurs identitaires reconnaissables tels que le locus, le code vestimentaire, les accessoires, les gestes et le discours.

Dans sa persona du policier-enquêteur, le personnage est représenté in situ, sur le lieu du crime, habillé en civil - informel mais à la mode - dont la seule marque de distinction est l'immanquable accessoire visuel de son identité professionnelle, son arme. Il est hyperactif : il court, il tire, il interroge et il procède à des arrestations; il se déplace en voiture de police traditionnelle mais aussi en $4 \times 4$, en hélicoptère ou en hors-bord. Il incarne, ainsi, l'homme ou la femme d'action, à l'image du héros traditionnel de la fiction d'aventure.

Dans sa persona du scientifique, le locus représentationnel se déplace vers le laboratoire ultramoderne. Comme le souligne Roberta Pearson dans son analyse de la série CSI, "Location inflects behaviour. [...] À great deal of the action in workplace dramas perforce takes place in a workplace that constructs character. [...] CSI staff spend a great deal of their time in stark and clinical labs which signal their unbiased and scientific professionalism.» (2007, p. 46)

ILCEA, 12 | 2010 
Dans cet environnement blanc et aseptisé, le policier-scientifique manipule des machines et des appareils mystérieux avec des écrans lumineux qui clignotent, qui calculent, qui mesurent, qui cherchent et qui trouvent, et tout cela sous les yeux médusés du spectateur:

There are lots of complicated looking laboratory instruments, complete with flashing lights, digital displays and multicoloured screens showing graphs and charts, all at the touch of a button. 'Let's run it through analysis,' says the police officer, handing the scientist a test tube of evidence that has just been scraped up from the crime scene or collected from the body being autopsied. The scientist dutifully plugs the sample straight into the machine, which beeps obligingly then, thirty seconds later, prints out the result. And not just any result. It's the critical clue that is going to identify the dangerous criminal." (Shelley Robinson 2007, p. 205)

Le changement de persona est aussi visuellement balisé par le biais d'un changement de code vestimentaire : en adéquation avec l'environnement technique au milieu duquel il évolue, le scientifique porte une blouse blanche immaculée pour afficher clairement son affiliation à la science.

Un autre marqueur identitaire qui met en relief la persona scientifique du protagoniste est le discours scientifique, un élément incontournable dans la construction du mythe de l'identité scientifique. Comme analysé ailleurs (Isani 2009b), tout ce qui fait référence au domaine scientifique - le factuel, le conceptuel, le technique, le fonctionnement et la manipulation des appareils, l'analyse des résultats, etc. - est présenté sous forme d'échanges orchestrés autour du tandem fictionnel du spécialiste qui parle à un nonspécialiste, ce qui permet de mettre en valeur à la fois le discours et les connaissances scientifiques de l'interlocuteur spécialisé. Cela donne lieu à la classique séquence discursive triptyque de vulgarisation (discours spécialisé + question naïve + discours de vulgarisation), comme l'illustre cet échange extrait du premier épisode de la première série des Experts Las Vegas (10/06/2000) où un entomologiste judiciaire et un nonspécialiste du domaine examinent les insectes sur un cadavre :

\begin{tabular}{|c|c|c|}
\hline $\begin{array}{l}\text { Discours } \\
\text { spécialisé }\end{array}$ & $\begin{array}{l}\text { Gil Grissom } \\
\text { (entomologiste) }\end{array}$ & «Pupa, stage three» \\
\hline Réponse naïve & $\begin{array}{l}\text { Jim Brass (non- } \\
\text { spécialiste) }\end{array}$ & " English! I'm not an entomologist » \\
\hline $\begin{array}{l}\text { Discours } \\
\text { vulgarisé }\end{array}$ & $\begin{array}{l}\text { Gil Grissom } \\
\text { (entomologiste) }\end{array}$ & $\begin{array}{l}\text { "It's the third stage of larva metamorphosis. This guy's } \\
\text { been dead for seven days." }\end{array}$ \\
\hline
\end{tabular}

57 Tous ces éléments, l'environnement, l'apparence, le discours, et bien entendu, le happy end, convergent pour représenter le scientifique comme un génie et la science comme un outil prodigieux. Contrairement à d'autres visions littéraires où la science est représentée comme une force de mal, destructrice de la société, comme par exemple Frankenstein ou le Prométhée moderne (1818) de Mary Shelley ou L'étrange cas de Dr Jekyll et Mr Hyde (1886) de R. L. Stevenson, dans le cas de la FASP criminalistique, la science est ici représentée comme une force positive et infaillible, protectrice de la société et en lutte contre le mal - une image qui s'inscrit dans la directe lignée des combats mythiques et bibliques entre les forces surnaturelles du mal et du bien. 


\section{Le médecin-légiste : de l'homme lugubre à la femme séduisante}

58 À l'instar du domaine scientifique dont il relève, le terme « médecin-légiste » présente une certaine instabilité terminologique. Dans la tradition de droit civiliste, le terme désigne le professionnel dont la fonction principale est de pratiquer des autopsies ${ }^{5}$. En revanche, dans la culture de common law, il existe des différences historiques, géographiques et juridictionnelles selon qu'il s'agit de la culture juridique britannique ou américaine, ce qui n'est pas sans engendrer une certaine confusion auprès du public. Dans le système juridique anglo-gallois, le coroner, qui possède une formation médicale ou juridique, dirige l'enquête qui doit déterminer la cause du décès, l'examen du corps in situ et l'autopsie relevant du domaine d'expertise du forensic pathologist. Aux États-Unis, le terme coroner est progressivement remplacé par celui de medical examiner-comme, par exemple, le protagoniste dans les romans de Patricia Cornwell - qui, tout en s'acquittant de certaines des fonctions administratives du coroner, est aussi celui qui examine le corps in situ et pratique les autopsies.

S'il existe quelques exemples récents de romans FASP portant sur le coroner - comme par exemple celui de M. R. Hall intitulé » The Coroner (2009) - c'est le médecin-légiste qui est le personnage de prédilection du genre. Dans son analyse de la mise en fiction de ce professionnel depuis 1903 dans Encyclopedia of Literature en ligne, Stephen Leadbeatter, lui-même spécialiste de la médecine légale, souligne la nature secondaire du rôle ("supporting role») attribué à ce personnage dans un genre où le rôle clé revient à l'enquêteur, au policier ou au détective: "The forensic pathologist is only one among the representatives of many disciplines and professions that make up the investigating team. [...] It should not be surprising therefore, that the forensic pathologist as protagonist looms small in detective novels and detective stories." Malgré cela, il convient de rappeler que bien que l'équipe d'experts judiciaires soit composée d'experts provenant de nombreuses disciplines diversifiées, le médecin-légiste est le seul professionnel de l'équipe à occuper un rôle récurrent - un fait lié sans doute à la fascination qu'a le public pour tout ce qui concerne la thanatologie.

60 La remontée d'intérêt pour la criminalistique et son évolution vers un sous-genre à part entière s'accompagne d'une profonde modification dans la représentation fictionnelle de ce professionnel. Dans les romans pré-FASP criminalistique, la mise en fiction du médecin-légiste est construite autour d'un personnage masculin excentrique, soit lugubre et austère soit jovialement macabre, dans la lignée de celui qui est dépeint dans Death of an Expert Witness:

Dr Reginald Blain-Thomson had a curious habit, before beginning his examination, of mincing round the body, eyes fixed on it with wary intensity as if half afraid that the corpse might spring into life and seize him by the throat. He minced now, immaculate in his grey pinstriped suit, the inevitable rose in its silver holder looking as fresh in his lapel as if it were a June blossom, newly plucked. He was a tall, lean-faced, aristocratic-looking bachelor with a skin as freshly pink and soft as that of a girl. He was never known to put on protective clothing before examining a body, and reminded Dalgliesh of those television cooks who prepare a four-course dinner in full evening dress for the pleasure of demonstrating their craft. It was even rumoured, unjustly, that Blain-Thomson performed his autopsy in a lounge suit. (1977, p. 262)

61 Avec l'arrivée de la FASP criminalistique, nous assistons à ce que Stephen Leadbeatter appelle "the renaissance of the forensic pathologist " marquée par un rôle plus soutenu et une représentation plus nuancée du médecin-légiste. 

représentation fictionnelle de cette profession jusqu'alors réservée exclusivement à la gente masculine. Deux protagonistes marquent particulièrement cette évolution. La première femme à être mise en fiction dans le rôle de médecin-légiste est sans doute le Dr Kay Scarpetta, medical examiner, qui fait son apparition dans ce qui est considéré comme le premier roman FASP criminalistique, Postmortem publié en 1990, un roman dont la popularité a été récompensée par l'attribution, dans la même année, des prix « Edgar ", «Anthony » et « Macavity » aux États-Unis, et du prix « Roman d'aventure » en France. A suivi en 1997 l'apparition d'une deuxième femme, Tempe Brennan, paléo-anthropologue judiciaire, avec la publication du roman Déjà Dead par Kathy Reichs. Contrairement au portrait fictionnel unidimensionnel du médecin-légiste masculin en tant que figurant, le personnage du protagoniste féminin dans la FASP criminalistique est présenté sous son aspect professionnel et privé : il s'agit d'une scientifique très compétente qui est aussi une femme belle, sportive, accomplie et séduisante, avec des affiliations familiales mais dont l'absence de mari permet d'entretenir des liaisons amoureuses d'un roman à l'autre.

observons la même tendance dans les séries télévisuelles où le médecine-légiste, bien qu'il n'occupe pas le devant de la scène comme protagoniste, est néanmoins l'un des personnages récurrents. Nous avons déjà eu l'occasion d'évoquer la jolie Dr Laura Hobson, médecin légiste dans la série Inspecteur Lewis, qui exerce son métier avec compassion pour les victimes qu'elle doit examiner et autopsier mais dont le statut de célibataire, combiné à celui du veuf de l'inspecteur Lewis, laisse présager des affinités extra-professionnelles.

Dans ce domaine, c'est sans doute le Dr Alexx Woods, médecin légiste de la série Les Experts Miami, qui étonne par la douceur et l'attention qu'elle apporte à « ses » cadavres. Belle, gracieuse et élégante, mère de deux filles, elle a l'habitude déconcertante - à la limite de l'excentricité ? - de s'adresser aux cadavres allongés sur sa table d'autopsie directement et affectueusement, les appelant sweetie, baby, honey, etc. Elle les manipule sans aucune manifestation de révulsion, les prenant dans ses bras dans une étreinte charnelle et maternelle. Pour le Dr Alexx Woods, cette tendresse est la meilleure façon de faire "parler» les cadavres car, d'après elle, à travers leur silence, les morts ont des choses à lui raconter. Cependant, en regard de cette tendresse inhabituelle envers les cadavres, se dessine en filigrane son obsession que ses interventions in situ vont peut-être la conduire à être, un jour, allongée comme ses « chéris » sur la table d'autopsie.

\section{Le cadavre : de réification à déification}

Le cadavre, malgré son silence et son immobilisme, est l'un des personnages incontournables de la fiction criminalistique, comme le souligne Ronald R. Thomas dans son ouvrage intitulé Dectective Fiction and the Rise of Forensic Science :

At the centre of virtually every detective story is a body upon which the literary detective focuses his gaze and employs his unique interpretive powers. His goal is to explain an event which seems to be inexplicable to everyone else. At stake is not just the identification of a dead victim or an unknown suspect, but the demonstration of the power invested in certain forensic devices embodied in the figure of the literary detective - the fingerprint, the mug shot, or the lie detector, for example - all of which enable the detective to read the clues to the mystery that is written in the suspect body. ([1999], 2003, p. 2)

Si le cadavre existe certes dans le roman policier, il est en général dépeint brièvement et in situ au début du roman, comme on peut le voir à travers cet extrait de Death of an Expert 
Witness, roman de P.D. James écrit presque deux décennies avant la publication du premier roman FASP :

In the front seat rested the body of a girl. Her legs were decorously planted together, the glazed eyes were slyly half open, the mouth, devoid of lipstick, was fixed in a drool elongated by two small trickles of blood. They gave a face which must have been pretty, or at least childishly vulnerable, the vacuous look of an adult clown. The thin coat, too thin, surely for a night in early November was pulled waist-high. $(1977$, p. 9)

Après cette description brève et retenue de l'élément déclencheur du récit policier, le cadavre ne réapparaîtra plus dans le récit qu'à titre d'évocation. En contraste, le roman à substrat ou à adstrat criminalistiques propulse le cadavre au-devant de la scène narrative et s'y attarde à travers des descriptifs graphiques, détaillés et répétés, comme l'illustre cet extrait de Death du Jour par Kathy Reichs :

Victim number one held its forearms high, flexed as if ready to fight. Pugilistic pose. While not long enough or hot enough to consume all the flesh, the fire had raced up the back wall and produced sufficient heat to cook the upper limbs and cause the muscles to contract. Below the elbows the arms were stick-thin. Clumps of scorched tissue clustered along the bones. The hands were blackened stumps.

The face reminded me of Rameses' mummy. The lips had burned away, exposing teeth with dark and cracked enamel. One incisor was delicately outlined in gold. The nose was burned and squashed, the nostrils pointing upward like the snout of a fruit bat. I could see individual muscle fibers circling the orbits and streaming across the cheekbones and mandible, like a line drawing in an anatomy text. Each socket had a dried and shriveled eyeball. The hair was gone. So was the top of the head. $(1999$, p. 27)

L'examen scientifique conduit inexorablement à la réification de l'objet d'observation, qu'il soit minéral ou organique. Ainsi, l'homme ou la femme qui animait jadis les sombres vestiges carbonisés décrits ci-dessus sera désormais réduit au statut d'un prélèvement, placé dans une boîte de Pétri, une pipette ou une boîte, identifié par une série de numéros - «Laboratoire de Médecine Légale number : 31013. Morgue number : 375. Police incident number: 89041.» (1999, p. 53) - et stocké quelque part dans un laboratoire :

It was late afternoon before the last of the bone was packaged and ready for transport. Ryan watched as I carefully extracted and wrapped the skull fragments and placed them in plastic containers. I would analyze the remains at the lab. (1999, p. 45)

69 La technique narrative contribue à renforcer le processus de réification lorsque, dans une approche comparable à la synecdoque, le cadavre est «débité " pour être réduit à la dimension d'une de ses composantes que le récit isole de l'entité et met en exergue, comme le démontre cet extrait du même roman :

The jaw. Gingerly, I teased away soil and ash to reveal a complete right ascending ramus, a fragment of the left ramus, and a portion of the mandibular body. The latter contained seven teeth. (1999, p. 39)

70 Dans la FASP criminalistique romanesque les processus scientifiques et les techniques narratives se conjuguent pour générer une représentation réifiée du cadavre. Les séries télévisuelles à substrat criminalistique ne suivent pas la même tendance. En analysant la façon dont est exploitée la puissance de la sémiotique visuelle, on constate deux principales orientations représentationnelles.

71 La première se situe dans une perspective d'humanisation: le cadavre est présenté en tant que personne individualisée et la caméra montre le visage et, parfois, le corps découverts. Le médecin-légiste lui adresse la parole directement, il le touche, le manipule et l'exhibe au spectateur fictionnel et réel, une approche très éloignée de la représentation visuelle du cadavre dans les romans policiers traditionnels où le visage du 
cadavre est montré brièvement et de loin, s'il n'est pas visualisé complètement couvert du linceul.

Une deuxième orientation représentationnelle, en revanche, poursuit le processus de déshumanisation du cadavre, mais en le sublimant en objet de déification. Les séries telles que CSI, NCIS, Inspecteur Lewis, Inspecteur Barnaby, etc., recourent à l'implicite de la mise en scène visuelle spécifique au medium pour représenter le cadavre comme un objet de culte : l'image visuelle récurrente est celle du cadavre allongé sur l'autel qu'est la table d'autopsie, tel un sacrifice offert aux dieux. Le lieu, cette salle d'autopsie que le récit narratif scriptural décrit froidement comme un laboratoire - "The autopsy room is small with a tile floor and the usual surgical cart, digital scale, evidence cabinet, autopsy saws and various blades, dissecting board and transportable autopsy table latched to the front of a wallmounted dissecting sink. ( (Cornwell, 2005, p. 42) - est visualisé dans les séries télévisuelles comme un lieu paisible et quasi onirique, très éloigné de la violence qui sous-tend son existence, comme le suggère Sue Turnbull : "The filters are blue. Grissom's coat is bluishwhite, the corpse is greyish-blue. The effect is dream-like. » (2007, p. 29) L'imaginaire perçoit ce lieu comme un sanctuaire, un espace sanctifié par cette chose sacrée qu'est le mystère de la mort et qui exige le respect de ceux qui s'y rendent pour contempler le martyr sacrificiel : on s'y rend en se couvrant entièrement de vêtements idoines, on écoute avec respect les « vérités » prononcées par le haut-prêtre des lieux qu'est le médecin-légiste, et on répond à voix basse.

Le cadavre, cette victime d'une mort violente, est présenté, souvent de profil, le visage serein et reposé, le corps partiellement couvert d'un linceul blanc, couleur de la pureté, ou vert pâle, couleur du calme. Le médecin-légiste et les enquêteurs, tous vêtus d'une tenue appropriée au lieu, encerclent l'« autel » et se vouent à réparer le tort commis à l'encontre de la justice de Dieu et des Hommes. Nous sommes, dans cette représentation visuelle, loin de l'image du cadavre d'une jeune fille coincée derrière le volant de sa voiture, le manteau relevé autour de la taille et les cuisses exposées, ou encore de celui carbonisé au point qu'il ne reste que quelques ossements pour témoigner de ce qui était jadis une vie humaine.

\section{Conclusion : perceptions du lectorat et « The CSI Effect "}

74 Après avoir analysé certaines des représentations projetées par la FASP criminalistique, il conviendrait d'aborder le pendant de cette question, les perceptions que de telles représentations forgent chez le lectorat. À ce sujet, il importe de souligner que s'il est communément admis que l'auteur de l'œuvre littéraire écrit pour lui-même, l'auteur de la fiction populaire écrit pour son lectorat. Se pose alors la question de savoir dans quelle mesure les attentes - et donc les perceptions - du lectorat façonnent et orientent l'écriture fictionnelle. Le sujet dépassant le cadre de cette étude, nous nous contenterons d'observer qu'il s'agit d'une question particulièrement sensible eu égard à la FASP où l'auteur spécialiste doit constamment veiller à calibrer le degré de spécialisation du substrat professionnel en fonction de l'évolution du degré de spécialisation de son lectorat.

75 Pour ce qui concerne la FASP criminalistique, de fortes représentations engendrent de fortes perceptions. Depuis l'an 2000, les observateurs ont pu identifier des manifestations 
comportementales et sociétales liées à des perceptions forgées par les représentations de la FASP criminalistique télévisuelle, un phénomène baptisé par conséquent, «the CSI effect » (d'après le titre en anglais de la série télévisuelle Les Experts), et qui se traduit par le vif engouement du grand public pour tout ce qui touche aux sciences criminalistiques.

Le premier reflet de cet engouement du public s'observe dans le domaine de la justice et du fonctionnement des jurys populaires, une institution plus répandue dans les pays relevant de la common law que dans les pays de tradition civiliste. Face à - ou à cause de la série Les Experts, tout téléspectateur assidu est désormais un expert en sciences criminalistiques, une tendance qui fait l'objet d'une mise en abîme métadiscursive dans Predator, FASP criminalistique par Patricia Cornwell, où le professionnel adresse ce même reproche à un collègue néophyte :

"I think you've been watching too many fantasy forensic shows. » Marino says, his big face staring at him. "Too much Harry Potter policing on your big flat-screen TV. Think you're a forensic pathologist, or almost one, a lawyer, a scientist, a crime scene investigator, a cop, Captain Kirk and the Easter Bunny all rolled up in one." (2005, p. 112)

L'homme lambda qu'est le juré populaire entretient désormais des attentes en matière de preuves et témoignages façonnées à l'image des représentations télévisuelles, une situation inquiétante d'après Michael D. Mann (2006) de la faculté de droit de l'université de Buffalo: «Through weekly television dramas discussing hyper-technical police procedures and legal proceedings, the criminal justice system has shifted into mainstream popular culture - a scary place since police and legal matters are presented to television viewers with no frame of reference.» (2006, p. 158) Armé d'une somme de connaissances souvent fragmentaires voire erronées, le juré souhaite aujourd'hui appuyer son «intime conviction » sur des preuves scientifiques. Ces exigences envers les preuves expertales ont des retombées sur les professionnels du droit qui se voient dans l'obligation d'une part de satisfaire à ces attentes et inversement, d'autre part, d'expliquer et corrriger les méprises qui en résultent, comme le résume Katherine Ramsland dans son ouvrage intitulé The C.S.I. Effect :

To be fair, there's something positive as well in the C.S.I. Effect. Increased jury awareness of the investigative process has helped to make it more accountable, and thus, investigators and evidence handlers are more conscious of what must be done to impress a jury. Nevertheless, the bar may be set so high at times - typically based on flawed ideas - that legal professionals despair. Few police departments have access to high-tech gadgets and expensive experts, so prosecutors find themselves at pains to de-educate juries, i.e., correct their misperceptions. (2006, XV)

Confrontés à ces exigences de preuves scientifiques, d'autres professionnels du droit n'hésiteraient pas à recourir à la preuve scientifique et au témoignage de l'expert judiciaire non par nécessité mais comme simple stratégie de communication destinée à mieux orienter la décision du jury.

Un deuxième effet de "l'effet CSI » relève du phénomène selon lequel les perceptions forgées à partir des représentations fictionnelles influent de manière importante sur des choix de carrière. Phénomène déjà documenté sous le nom de " the Perry Mason syndrome " ou encore dans le cadre de la série de FASP médicale ER, nous l'observons en lien avec la FASP criminalistique à présent. Le mythe de la science infaillible comme force de bien attire les jeunes et l'un des effets mesurables en est l'augmentation du nombre d'inscriptions dans les facultés des sciences en vue de devenir "expert judicaire », un métier perçu comme étant " a cool job to have " selon les témoignages de deux professionnels, Daryl Vinall et Shelley Robinson (2007, p. 202). 
80 Dans le cadre des sciences criminalistiques, ce phénomène a été identifié dans les universités du Royaume-Uni, des États-Unis et de l'Europe continentale. À cet égard, les statistiques publiées par l'École des Sciences Criminelles de Lausanne témoignent de l'explosion des inscriptions en 2001, un an après la diffusion de la série Les Experts, une tendance en augmentation croissante entre 2001 et 2007, représentant une hausse globale de $94 \%$ :

Tableau 1. Inscriptions à l'École des sciences criminelles, Lausanne (Suisse)

\begin{tabular}{|l|c|c|c|}
\hline & 1995 & 2001 & 2007 \\
\hline Inscriptions $1^{\text {re }}$ année & 117 & 221 & 426 \\
\hline \hline Diplômés & 15 & 34 & 78 \\
\hline
\end{tabular}

(Sources : <http://sciencesforensiques.com>)

81 Cependant, comme on ne manquera pas de le remarquer, le tableau indique également que si, en 2007, 426 jeunes se sont inscrits en $1^{\text {re }}$ année, seulement 78 ont été diplômés. Entre le rêve engendré par la fiction et la réalité souvent fastidieuse des sciences criminalistiques, il existe un fossé, comme le soulignent Daryl Vinall et Shelley Robinson :

The main problem is that, as in all dramatisations, it is the tedious side of the job that is left out. Meticulous calculation of the gap between furniture and walls, measuring of evidence in relation to walls and furniture, exact specifications of a room and where the exit and entry points are hardly riveting TV. [...] The job is in fact, nowhere as glamorous as it seems. As is always in television drama most of the mundane side of life is left out. (2007, p. 203)

\section{BIBLIOGRAPHIE}

BIROS, Camille, «Quand l'impensable surgit dans le réel - première cartographie d'un type émergeant de FASP : l'écothriller ", ASp la revue du GERAS, n 57, 2010, p. 67-85.

CHARPY, Jean-Pierre, «FASP médicale et substrat professionnel : le miroir éclaté », ASp la revue du GERAS, ${ }^{\circ}$ 57, 2010, p. 49-63.

DUBoIs, Jean, Mathée GIACOMo, Louis GUESPIN, Christiane MARCELLESI, Jean-Baptiste MARCELLESI, Jean-Pierre MÉVEL, Dictionnaire de linguistique et des sciences du langage, Paris, Larousse. 1994.

FOMBONNE, Jacques, La criminalistique, Paris, PUF, 1996.

ISANI, Shaeda, « From idealisation to demonisation \& in-between : representations of American lawyers in legal FASP », ASp, n 47-48, 2005, p. 67-81.

ISANI, Shaeda, 2009a, «Journalism FASP \& fictional representations of journalists in popular contemporary literature ». Langues \& cultures de spécialité à l'épreuve des medias / Specialised 
discourse and culture through the prism of the media. Isani, Shaeda (dir), [En ligne] <http:// ilcea.revue.org>.

ISANI, Shaeda, 2009b, « Specialised fictional narrative and lay readership : bridging the accessibility gap ». ASp, $\mathrm{n}^{\circ}$ 56, 2009/2, p. 45-65.

ISANI, Shaeda, « English for Legal Purposes \& Domain-specific Cultural Awareness : the 'Continental Paradox', definition, causes \& evolution ", Amsterdam, à paraître en 2010, John Benjamins.

ISANI, Shaeda, «Bench \& Bar in Popular Legal Fiction : A Comparative Approach to Fictional Representations \& Public Perceptions ", GRAAT On-line \# 7, p. 182-200. [En ligne] <http:// www.graat.fr/j-isani.pdf>, 2010.

LITTLE, William, H. W. Fowler \& Jessie Coulson (eds), The Shorter Oxford English Dictionary on Historical Principles, Oxford, Clarendon Press, 1973.

MALMKJAER, Kirsten (ed.), The Linguistics Encyclopedia, London, Routledge, 2004.

MANN, Michael D, “The 'CSI Effect' : Better Jurors through Television and Science ?", Buffalo Public Interest Law Journal, 2006, vol. XXIV, p. 157-183.

MAZÉVET, Michel, Edmond Locard: Le Sherlock Holmes français, Lyon, Editions des Traboules, 2006. NICHOLSON, Kate \& Anne Stevenson (dir.), Dictionnaire Juridique / Law Dictionary. Edinburgh / Paris, Harrap's Dalloz, 2004.

PEARSON, Roberta, « Anatomising Gilbert Grissom: The Structure and Function of the Televisual Character » dans Reading CSI: Crime TV Under the Microscope (ed), Michael Allen, Londres, I. B. Tauris, 2007.

PESNOT, Patrick, Les Détectives de l'impossible, Paris, Denoël. 1999.

PETIT, Michel, « La fiction à substrat professionnel : une autre voie d'accès à l'anglais de spécialité », ASp n²3/26, 1999, p. 57-81.

REY-DEBOVE, Josette \& Alain REY, Le Petit Robert, Dictionnaire de la langue française, Paris, Dictionnaires Le Robert, 2003.

THOMAS, Ronald R., Detective Fiction and the Rise of Forensic Science, Cambridge, CUP, 1999. TURNBULL, Sue, "The Hook and the Look: CSI and the Aesthetics of the Television Crime Series » dans Reading CSI: Crime TV Under the Microscope, Michael Allen (ed.), Londres, I. B. Tauris. 2007. VAN DER YEUGHT, Michel,Une histoire de Wall Street, Paris, Editions ESKA, 2009.

VINALL, Daryl \& SHELlEY Robinson, « Dissecting CSI: The View from the Trainee and the Professional » in Reading CSI : Crime TV Under the Microscope, Michael Allen (ed.), London, I. B. Tauris, 2007.

\section{Sitographie}

Sciences Forensiques [En ligne], <http://sciencesforensiques.com>, consulté le 04/04/2009. Criminalistique. Encyclopédie Universalis <http://www.universalis.fr>, consulté le 22/06/2009. World of Forensic Science / Criminalistics. [En ligne], http://www.enotes.com/forensic-science/ criminalistics/print, consulté le 18/06/2009.

Forensic : Confédération suisse, Chancellerie fédérale : [En ligne], <http://www.bk.admin.ch/...>, consulté le 04/05/2009. 
Leadbeatter, Stephen. « Forensic Pathologist : Black and White, The Romance of Poisons, The Red Thumb Mark, Sleuths, Regina, Gummer ». Encyclopedia of Literature. [En ligne], <http:// www.jrank.org/literature/...>, consulté le 16/12/2009.

\section{Corpus}

\section{Références romanesques}

BEGLEY, Louis, About Schmidt, New York, Ballantine Books, 1996.

CONAN DOYLE, Arthur, À Study in Scarlet, London, Ward Lock \& Co, 1887.

cook, Robin, Crisis, New York, Putnam, 2006.

CORnWELl, Patricia, Postmortem, Londres, Sphere, 1990.

CORNWELl, Patricia, Predator, Londres, Sphere, 2005.

DEAVER, Jeffrey, The Bone Collector, New York, Signet, 1998.

GRISHAM, John, A Time to Kill, Londres, Arrow Books, 1989.

GRISHAM, John, The Last Juror, Londres, Century, 2004.

HALL, M. R., The Coroner, Londres, Macmillan, 2009.

JAMES, P. D., Death of an Expert Witness, Londres, Faber and Faber, 1977.

LARSSON, Stieg, Millenium, traduit du suédois par Lena Grumbach et Marc de Gouvenain. Paris, Actes Sud, 2007.

MARKLUND, Liza, [2002] The Bomber, traduit du suédois par Kajsa von Hofsten, Londres, Pocket Books, 1998.

MARTINI, Steve, Double Tap, Londres, Putnam, 2005.

MINA, Denise, The Field of Blood, Londres, Bantam, 2005.

REICHS, Kathy, 206 Bones, New York, Scribner, 2009.

REICHS, Kathy, Death du Jour, Londres, Arrow Books, 1999.

WOLfE, Tom, The Bonfire of the Vanities, Londres, Picador, 1987.

\section{Références télévisuelles}

Bones, producteur Kathy Reichs, septembre 2005 jusqu'à aujourd'hui.

CSI Las Vegas, producteur Jerry Bruckheimer, octobre 2000 jusqu'à aujourd'hui.

CSI Manhattan (CSI New York), producteur Jerry Bruckheimer, septembre 2004 jusqu'à aujourd'hui.

CSI Miami, producteur Jerry Bruckheimer, septembre 2002 jusqu'à aujourd'hui.

Inspecteur Barnaby (Midsomer Murders), producteurs Betty Willingale et Brian True-May, mars 1997 jusqu'à aujourd'hui.

Inspecteur Lewis (Lewis), auteurs Colin Dexter et Russel Lewis, réalisateur Bill Anderson, mars 2007 jusqu'à aujourd'hui.

Inspector Morse, auteur Colin Dexter, producteur David Lascelles, 1987-2000.

NCIS, Producteur Donald P. Bellisario et Don McGill, septembre 2003 jusqu'à aujourd'hui. 


\section{NOTES}

1. Le terme «adstrat» provient du domaine de la linguistique et est défini comme suit par Dubois et alii : "On donne le nom d'adstrat à la langue ou au dialecte parlé dans une région voisine du pays où on parle la langue prise comme référence.» $(1994$, p. 19). Pris dans cette acception, le terme véhicule ainsi le caractère de secondarité qui est celui de l'adstrat professionnel par rapport au substrat professionnel dominant.

2. Les termes Forensic sciences ou forensics proviennent du latin forensis signifiant «forum », c'està-dire la place publique où se discutait les affaires publiques et où se tenait les procès de justice dans l'Antiquité.

3. Quelques exemples en ligne: < http://www.lcl.ne.us/depts/...> ; <http:// www.overbooked.org/...> ; <http://www.lib.il.us/...>

4. À ce sujet, il est à se demander si ce n'est pas la réalité qui influe sur la fiction. Aux États-Unis où la télévision est souvent autorisée à diffuser le déroulement du procès en direct, le monde entier a pu voir défiler les témoins experts à la barre dans le procès d'O. J. Simpson en 1994.

5. À la lumière de cela, l'observation fictionnelle de Kathy Reichs relative aux fonctions du médecin-légiste en France soulève quelques interrogations : «In France, pathologists do everything, anthropology, odontology, whatever. It's an archaic approach to forensic medicine, but there you have it. » (206 Bones, 2009, p. 274-275)

\section{RÉSUMÉS}

Après une brève introduction à la fiction à substrat professionnel (FASP), l'article propose de situer le domaine méconnu des sciences criminalistiques dans les cultures de common law et de tradition civiliste et en passant, évoque les problèmes de traduction anglais-français existants à cet égard. Il traite ensuite de l'émergence de ce nouveau sous-genre de la FASP qu'est la FASP criminalistique et la glorification de la science qu'il véhicule à partir des romans de Patricia Cornwell, Kathy Reichs et des séries télévisuelles Les Experts, etc.

L'article aborde ensuite les représentations fictionnelles que la FASP criminalistique génère des scientifiques et de leur environnement professionnel à partir de l'analyse de trois personnages clés du genre, la police scientifique, le médecin-légiste et le cadavre.

En guise de conclusion, l'article présente une brève analyse des perceptions que de telles représentations de la science engendrent notamment dans les domaines de la justice et de l'université.

After a brief introduction to a recently identified genre of popular literature, fiction à substrat professionnel or FASP, the article situates the relatively unfamiliar domain of forensic sciences in common and civil law cultures, and draws attention to French-English translation problems existing in this field. The article then analyses the emergence of a new FASP sub-genre, forensic FASP and its characteristic glorification of the sciences as illustrated by the novels of Patricia Cornwell and Kathy Reichs and TV series such as CSI.

It next goes on to explore the fictional representations that forensic FASP generates of forensic experts and their professional environment by analysing three main protagonists of the genre, 
the forensic expert, the forensic pathologist and the cadaver.

To conclude, the article presents a brief analysis of the consequences of perceptions shaped by fictional representations of forensic sciences with reference to such domains as those of justice and academia.

INDEX

Keywords : FASP, specialised fiction, forensic fiction, scientific thrillers, fictional representations, forensic experts, medical examiner, forensic pathologist, CSI, Patricia Cornwell, Kathy Reichs

Mots-clés : FASP, fiction, criminalistique, thriller scientifique, représentations fictionnelles, police scientifique, médecin-légiste, Les Experts, Patricia Cornwell, Kathy Reichs 